\title{
Síntomas de depresión en hombres
}

\section{Depression Men's Symptoms}

\author{
Constanza Londoño PÉRez \\ Universidad Católica de Colombia, Colombia \\ ORCID: http://orcid.org/0000-0003-3273-3658 \\ Wenceslao Peñate Castro \\ Universidad de La Laguna, España \\ Manuel GonzÁlez Rodríguez \\ Universidad de La Laguna, España
}

a Autor de correspondencia. Correo electrónico:
clondono@ucatolica.edu.co

Para citar este artículo: Londoño Pérez, C., Peñate Castro, W., \& González Rodríguez, M. (2017). Síntomas de depresión en hombres. Universitas Psychologica, 16(4), 1-19. https://doi.org/10.11144/Jav eriana.upsy16-4.sdeh

\section{RESUMEN}

El objetivo de este estudio era el objetivo de la presente investigación comparativa es identificar los síntomas específicos de depresión en hombres y determinar si existe un sesgo diagnóstico en los criterios incluidos en el sistema DSM y CIE. Para ello se estudia una muestra clínica conformada por 156 hombres y 174 mujeres entre los 18 y los 65 años, con diagnóstico previo de depresión, y un grupo control compuesto por 149 hombres y 151 mujeres sin depresión. Para evaluar depresión se usó Cuestionario de Depresión en Hombres (CDH) y la Entrevista MINI (Mini International Neuropsychiatric Interview). Para los análisis psicométricos se empleó el Modelo de Rasch desde la Teoría de Respuesta al Ítem y análisis comparativos multivariados. Se concluye que los hombres se deprimen de forma significativamente diferente de las mujeres y que parte de los síntomas identificados no son considerados en los sistemas nosológicos vigentes.

\section{Palabras clave}

depresión; hombres; masculinidad; síntomas.

\section{ABSTRACT}

The aim of this study was to identify if there are symptoms of depression in men who are not described in diagnostic manuals available. This clinical sample comprised 156 men and 174 women between 18 and 65 years prior to diagnosis of depression, and 149 men and 151 women without depression used as controls is studied. To evaluate Depression Questionnaire Depression in Men (CDH) and Interview MINI (Mini International Neuropsychiatric Interview) was used. For psychometric analyzes the Rasch model was used from the Item Response Theory and multivariate comparative analysis. We conclude that men are depressed significantly different from women and that some of the symptoms identified are not considered in the current nosological systems.

Keywords

Depression; men; masculinity; symptoms.

Cerca de 350 millones de personas padecen depresión en el mundo (Organización Panamericana de la Salud [OPS], 
2012), con una proyección poco alentadora de que hacia el 2030 sea la primera causa de morbilidad en mujeres y haya aumentado considerablemente en hombres (Organización Mundial de la Salud [OMS], 2009). El impacto que tiene este trastorno sobre la salud y la productividad ha motivado múltiples investigaciones, aunque aún es necesario develar importantes interrogantes respecto de los mecanismos causales, las características clínicas universales del trastorno, sus expresiones particulares en grupos específicos y sus límites diagnósticos con ansiedad, duelo y algunos problemas mentales del espectro psicótico.

De acuerdo con el DSM y el CIE hombres y mujeres se deprimen de la misma forma, ya que en ambos sistemas solo se reconocen diferencias en los síntomas de depresión reportados por niños y adolescentes, aunque las expresiones dependientes del sexo pueden ser cualitativamente distintas. Es conocido que mujeres jóvenes deprimidas parecen ser más propensas a mostrar autovaloración y rumiación negativas, síntomas somáticos, rumiación, llanto frecuente y mayor insatisfacción con la imagen corporal que los hombres deprimidos; por su parte, los hombres expresan más inhibición en el trabajo, fatiga y problemas de salud (Bennett, Ambrosini, Kudes, Metz, \& Ravinovich, 2005; Franchi, 2001).

Las diferencias en los síntomas de depresión existentes entre hombres y mujeres parecen persistir a través de las culturas, muestra de ello son los resultados obtenidos en la Encuesta São Paulo Megacity de Salud Mental (Viana \& Andrade, 2012), en el estudio alemán, en una muestra de 4075 casos y el estudio trasnacional de 15 países realizado en Europa en 2010 (Van de Velde, Bracke, \& Levecque, 2010). Es importante hacer notar que en general las mujeres tienen mayor capacidad que los hombres para recordar y hacer recuentos retrospectivos de síntomas, ello facilita que las mujeres alcancen más fácilmente umbrales diagnósticos.

Dichas investigaciones indican diferencias en la severidad y agudeza de los síntomas, con mayor frecuencia de depresiones bipolares, e inicios más tempranos, alta recurrencia en mujeres, y mayor duración con menor fluctuación del estado de ánimo y baja recurrencia debida a que son poco frecuentes las curvas de recuperación (Lucht et al., 2003; Wang, Hsu, Chiu, \& Liang, 2012). No obstante, y a pesar de que parece ser que las mujeres tienen depresiones más agudas, es necesario señalar que la depresión masculina es una de las principales causas de suicidio en el mundo (Haeffel et al., 2007; Instituto Nacional de Medicina Legal y Forense, 2011; Merikangas et al., 2011; Mittendorfer-Rutz, 2006; Troister \& Holden, 2010).

Diversos autores han observado que existe una seria diferencia en la forma cómo expresan la depresión hombres y mujeres (Brownhill, Wilhelm, Barclay, \& Schmied, 2005; Cochran \& Rabinowitz, 2003; Möller-Leimkühler \& Yücel, 2010; Strömberg, Backlund, \& Löfvander, 2010). Es así como, las mujeres se caracterizan por tener cuadros depresivos altamente melancólicos, subvaloración personal, insatisfacción con la imagen corporal, rumiación negativa, incremento del apetito y del peso, problemas de concentración y llanto frecuente (Essau, Lewinsohn, Seeley, \& Sasagawa, 2010); por el contrario, los hombres muestran retraso en la activación psicomotora, menosprecio de su capacidad personal, fatiga, problemas de salud (Bennett et al., 2005), más frecuente reporte de síntomas bipolares como disminución del apetito, ideación suicida, aislamiento social, sensación de ser visto por otros como inadecuado o en proceso de deterioro, que denotan alta sensibilidad al rechazo (Viana \& Andrade, 2012; Wenzel, Streer, $\&$ Beck, 2005).

Respecto del curso de la enfermedad, si bien las mujeres presentan depresiones con episodios más largos, mayor número de ocurrencias y más altas recurrencias, los hombres tienen episodios de inicio más temprano, con menor fluctuación del estado de ánimo y menor recurrencia debida a baja recuperación, ya que su estado se hace crónico con enriquecimiento y agudización de los síntomas somáticos (Essau et al., 2010; Lucht et al., 2003; Van de Velde et al., 2010; Viana \& Andrade, 2012). Para otros autores, los hombres tienen depresiones de mayor duración asociada a la alta comorbilidad con consumo prolongado de 
sustancias que a su vez incrementa el deterioro de los síntomas depresivos (Essau et al., 2010).

Desde los años 80, se ha evidenciado un aumento sostenido de casos diagnosticados con algún tipo de depresión, aun cuando parece existir sesgo en el DSM-IV-TR y en el reciente DSM V (American Psyquiatric Association [APA], 2002, 2013), debido a que los estudios tipo que dieron origen a la lista sintomatológica del trastorno han sido conducidos principalmente con muestras compuestas por mujeres (Álvarez \& Londoño, 2012), ya sea porque acuden más frecuentemente a los servicios sanitarios o participan más de los estudios tomados como base para definir los criterios diagnósticos, y las estrategias de evaluación válidas disponibles se derivan del sistema nosológico vigente. Sin duda, las mujeres tienden a buscar más soporte profesional (Matud-Aznar, 2008) y son más expresivas emocionalmente. De allí, el probable subdiagnóstico en hombres y la necesidad de estudiar posibles diferencias en la cronicidad y recurrencia de los síntomas.

Pareciera que el valor evolutivo de la tristeza, que es disminuir la activación para incrementar la reevaluación, permitir el análisis de las situaciones que le provocan inquietud y comunicar la necesidad de apoyo (Clore \& Colcombe, 2003; Niemeyer, 2006), en los hombres se pierde ya que el procesamiento es sesgado por esquemas de pensamiento referidos al rol social de autonomía, liderazgo, invulnerabilidad, fortaleza, protección y a los valores sociales que median los juicios acerca de la valía personal. Estos sesgos le indican al hombre que debe actuar de inmediato, sin dar lugar a la experiencia sensible necesaria para generar conciencia del problema y las emociones asociadas, ya que eso le resta estatus (Ordaz \& Luna, 2012).

Entonces, la tendencia a reconfirmar los esquemas negativos de autonomía y sociotropía, enfocados en resaltar la importancia de la independencia (Zapata \& Giner, 2009), centrados en la necesidad de privacidad, autodeterminación y libertad (Hankin, 2010; Liu \& Alloy, 2010), hacen que los hombres actúen para responder a las demandas sociales de perfección, independencia, autodeterminación, productividad (Jahromi, Naziri, \& Barzegar, 2012), poder y control social (van de Boogaard, Verhaak, van Dyck, \& Spinhoven, 2011). Por ello, el hombre debe resolver, en su totalidad o en una buena parte, las necesidades económicas, de supervivencia y seguridad de su grupo familiar (Galea et al., 2007), aun en tiempos de crisis social y económica (Moore \& Hadjiyannakis, 2002; Palomar \& Cienfuegos, 2007). De lo contrario, aumenta su percepción de amenaza que disminuye su confianza personal (Juárez \& Guerra, 2011) y facilita la aparición de cuadros de depresión.

Adicionalmente, el rol masculino enfrenta a los hombres a situaciones en las que tienen que tomar decisiones en asuntos cruciales que afectan a otros o representan cambios en el rumbo de la vida, previamente asociados a la aparición y mantenimiento de cuadros depresivos (Mossakowski, 2008). Es tan fuerte este efecto de la masculinidad, que en sociedades altamente machistas, los hombres que pierden sus bienes, su rol social de mando, consideran que fallaron en la protección de su familia, no responden sexualmente con vigor, prefieren morir antes de sentirse deshonrados (Adinkrah, 2012; Branney \& White, 2008; Broom \& Tovey, 2009).

En general, los síntomas de depresión masculina son rechazados socialmente pues son considerados propios de la vulnerabilidad emocional femenina, debido a esto los hombres se encuentran menos dirigidos a reconocer que están tristes y desesperanzados debido a los problemas que afrontan, prefieren aducir estrés o exceso de trabajo para explicar la preocupación y el llanto. Desde esta perspectiva machista, los varones deben dominar, competir, tener fuerza corporal y soportar estoicamente el dolor; por el contrario, la depresión los hace sentirse débiles, cansados, sin energía y con la necesidad de soporte profesional estrechamente asociado al rol femenino (Emslie, Ridge, Ziebland, \& Hunt, 2006; Kilmartin, 2005; Möller, Heller, \& Paulus, 2006; Rihmer, Rutz, \& Pihlgren, 1995).

Para Carvalho y Hopko (2011), la depresión en los hombres está asociada a la tendencia 
masculina a la evitación cognitiva, emocional y conductual que facilita la acumulación de tensión, por ello los síntomas son indirectos y difíciles de detectar. Los hombres parecen tener una predisposición a inhibir emociones displacenteras a través de la búsqueda de sensaciones extremas como volcarse en el trabajo, abusar de drogas, tener sexo sin control o cometer delitos (Angst, Gastpar, Lépine, Mendlewicz, \& Tylee, 2002; Beutel, Glaesmer, Wiltink, Marian, \& Brahler, 2010; Fuss et al., 2010; Hölzel, Härter, Reese, \& Kriston, 2011; McCusker \& Galupo, 2011; Strömberg et al., 2010).

El incremento de la tensión emocional, asociado a esta tendencia evitativa, puede enlazarse con la hostilidad, ira y agresividad característicos de la depresión mayor en hombres, ya que las activaciones del sistema de alerta ante situaciones amenazantes provocan reacciones de lucha o huida. Para Möller et al. (2006) y Möller-Leimkühler (2002), la exacerbación de la agresividad nace de la baja tolerancia al estrés, el pobre control de los impulsos, la inquietud y la insatisfacción.

En los hombres, los síntomas de depresión aparecen de forma gradual, con un patrón de ocurrencia que se inicia con aumento de la tensión emocional, seguido del incremento de la conducta agresiva y se cierra con el abuso de drogas (Bech, Olsen, Kjoller, \& Ramussen, 2003). La conducta agresiva en hombres deprimidos se expresa en el aumento de hostilidad no verbal (Fava, Nolan, Kradin, \& Rosenbaum, 1995; Katz, Wetzler, \& Cloitre, 1993) y la aparición de comportamiento autodestructivo como abuso de drogas y realización de actividades de alta peligrosidad (Rutz, 2004); ambas conductas son tenidas en cuenta en el sistema diagnóstico, solo en casos de intento suicida.

La exacerbación de los síntomas agresivos puede asociarse a la frustración derivada del incumplimiento el encargo social de cumplir metas laborales y académicas, protegerse y proteger a otros (Emslie et al., 2006; Levola , Holopaine, \& Aalto, 2010). Cabe notar que la conducta agresiva, frecuente en casos de depresión infantojuvenil, puede deberse a falta de maduración emocional, pero esta afirmación es altamente discutible. En la misma línea, Wai y Tse (2009) y Wai, Jayne y KaiChung (2011) evidenciaron que quienes guardan hostilidad y rencor obtienen menor soporte social y desarrollan más frecuentemente depresión mayor.

De otra parte, los hombres deprimidos tienen mayor frecuencia de conducta suicida efectiva, probablemente por no detección oportuna del problema o porque frecuentemente rehúsan la asistencia profesional (Mittendorfer-Rutz, Rasmussen, \& Wasserman, 2004). Si la detección de los casos de depresión está siendo ineficiente, es urgente desarrollar estrategias válidas y confiables para tamizar los casos y ofrecer atención oportuna para evitar la agudización de los síntomas.

En resumen, entre los síntomas de depresión masculina están: reducido autodominio (cólera crónica, alta hostilidad y agresividad), irritabilidad creciente y cambios bruscos del estado de ánimo; conductas autolesivas (abuso de drogas, exposición voluntaria al riesgo, promiscuidad); conductas evitativas (exceso de trabajo, fatiga, desinterés, negación del problema, distanciamiento social e intentos suicidas en casos agudos); subvaloración personal (baja autoeficacia, sensación permanente de inutilidad), menosprecio (devaluación personal y alta autocritica), sensación de empeoramiento de la imagen social, sensación de desamparo, desesperación, dificultad para resolver problemas y sensación de estar vacío.

Saber cuáles son los síntomas característicos de depresión en hombres podría aportar en el diagnóstico efectivo y oportuno, en la prevención del suicidio y en el desarrollo de intervenciones específicas efectivas.

\section{Método}

Diseño

El presente estudio descriptivo comparativo tuvo como objetivo identificar los síntomas específicos 
de depresión en hombres y determinar si existe un sesgo diagnóstico en los criterios incluidos en el sistema DSM y CIE.

Muestra

La muestra estuvo conformada por 630 personas entre 18 y 64 años (media de 28.04 y desviación estándar de 11.02), de nivel socioeconómico medio-bajo, con nivel educativo mínimo de primaria, pero principalmente profesional que acudieron a consulta por diversos motivos entre los meses de mayo y noviembre de 2013 a centros de salud ubicados en cinco ciudades principales colombianas (Bogotá, Medellín, Bucaramanga, Ibagué y Cali). Para llevar a cabo la comparación de síntomas, se agruparon teniendo en cuenta el género y la condición clínica: el primer grupo (G1) estaba conformado por 149 hombres sin depresión, el segundo (G2) por 151 mujeres sin depresión, el tercero (G3) por 174 mujeres diagnosticadas con depresión y el cuarto (G4) por 156 hombres diagnosticados con depresión.

Entre los criterios de inclusión para los grupos G3 y G4 estaban: 1) existencia de diagnóstico depresión realizado por un profesional y 2) no inicio de tratamiento. En los grupos no clínicos: 1) que en la Entrevista MINI de Depresión (Sheehan et al., 1998) y en el Cuestionario de Síntomas Autorreportado SQR no mostraran indicadores clínicos, y 2) que sus características sociodemográficas (género, edad, nivel educativo y estrato socioeconómico) fueran similares a las de los grupos clínicos.

\section{Instrumentos}

Cuestionario de Depresión en Hombres ([CDH]; Álvarez \& Londoño, 2011)

Modificado por Londoño, Peñate y González (2016), el cuestionario tiene como objetivo identificar síntomas característicos de depresión en hombres y está compuesto por 40 ítems divididos en 6 factores: Autoimagen negativa, Pobre imagen social, Afecto negativo, Ideación suicida, Desesperanza y Evitación; además, permite determinar el nivel de severidad de la depresión, si es el caso; tiene cuatro opciones de respuesta en escala Likert de frecuencia de siempre a nunca. La fiabilidad por factor es alta y oscila entre 0.75 y 0.93 , con una fiabilidad global de 0.9.

Ficha de información sociodemográfica

Formato de registro que recoge información como: género, edad, nivel educativo, procedencia, estado civil, información laboral (horas al día dedicadas, ocupación, nivel de satisfacción con la labor realizada), actividad deportiva o física (horas al día dedicadas) y estrato socioeconómico.

Entrevista Neuropsiquiátrica Internacional MINI (Mini International Neuropsychiatric Interview)

Utilizada como estrategia de selección para los casos incluidos en los grupos no clínicos, en las escalas relacionadas con trastornos del estado de ánimo (escalas de episodio depresivo mayor, trastorno distímico y riesgo de suicidio). El MINI ha sido usado como entrevista diagnóstica estructurada de breve duración que explora de manera independiente los principales trastornos psicopatológicos del eje I del DMS IV y el CIE-10. Tiene un alto nivel de discriminación clínica y la confiabilidad test-retest está entre 0.76 y 0.93 (Sheehan et al., 1998). La Entrevista, en su apartado de depresión, incluye un Factor 1 de Severidad de la depresión actual conformado por las preguntas 1 a 12, un Factor 2 de Distimia conformado por las preguntas 13 a 27 y el Factor 4 de Ideación suicida que incluye las preguntas 28 a la 32.

Previo consentimiento informado, las entrevistas fueron aplicadas por profesionales psicólogos o psiquiatras que se encontraban estudiando un postgrado sin que se diera interacción entre el profesional remisor y el entrevistador para evitar sesgos en las entrevistas reconfirmatorias del diagnóstico. El análisis de resultados incluyó estadísticos descriptivos, análisis comparativos ANOVA con el paquete SPSS y análisis de funcionamiento diferencial de los ítems realizados bajo el modelo de Rasch con el paquete estadístico SPAD. 


\section{Resultados}

Se compararon las medias de los puntajes estandarizados obtenidos por hombres y mujeres, y se encontró que no existía diferencia entre los grupos G1 y G2 (grupos no clínicos); con diferencias significativas $(p \leq 0.05)$ entre los grupos no clínicos y los clínicos (G3 y G4). También se encontraron diferencias significativas de los puntajes totales del CDH entre los grupos clínicos de hombres y mujeres (G3 y G4). El grupo de hombres deprimidos (G4) alcanzó los promedios más altos en el $\mathrm{CDH}$, pero el grupo de mujeres deprimidas (G3) presentó la mayor variabilidad en las respuestas dadas a dicho cuestionario (Tabla 1).

\section{TABLA 1}

Comparación de medias del puntaje total obtenidas en el Cuestionario de Depresión en Hombres entre los grupos

\begin{tabular}{cccccccc}
\hline \multirow{2}{*}{ Cuestionario } & \multicolumn{2}{c}{ Grupos Comparados } & \multicolumn{3}{c}{ Diferencia } & \multicolumn{3}{c}{ Welch -2 colas } \\
\cline { 2 - 8 } & Grupo & Grupo & Media & $D E$ & $t$ & DIF & $p$ \\
\hline & $1(\mathrm{n}=149)$ & $2(\mathrm{n}=151)$ & 0.35 & 0.29 & 1.13 & 88 & 0.26 \\
$\begin{array}{c}\text { Cuestionario de } \\
\text { Depresión en } \\
\text { Hombres }\end{array}$ & $1(\mathrm{n}=149)$ & $3(\mathrm{n}=174)$ & -1.18 & 0.29 & -4.13 & 83 & 0 \\
& $1(\mathrm{n}=149)$ & $4(\mathrm{n}=156)$ & -1.4 & 0.2 & -7.09 & 110 & 0 \\
& $2(\mathrm{n}=151)$ & $3(\mathrm{n}=174)$ & -1.51 & 0.32 & -4.29 & 111 & 0 \\
& $2(\mathrm{n}=151)$ & $4(\mathrm{n}=156)$ & -1.75 & 0.29 & -5.96 & 90 & 0 \\
& $3(\mathrm{n}=174)$ & $4(\mathrm{n}=156)$ & -0.25 & 0.28 & -0.83 & 84 & 0 \\
\hline
\end{tabular}

Nota. p: Nivel de significancia menor o igual a 0.05; DE: Desviación estándar; DIF: Diferencia; $t$ : $t$ de Student. Fuente: elaboración propia.

En el análisis de funcionamiento diferencial de ítems respecto del género se contrastan las posibles diferencias teniendo en cuenta que: a) el valor de la diferencia (Difcontrast) fuera mayor a 0.5 ; b) que la probabilidad de Welch inferior a $0.5 \mathrm{y} \mathrm{c}$ ) que la probabilidad Mantel Haenzel fuera inferior a 0.05 (Tabla 2).

Se encontró que los ítems 12, 17, 28, 30 y 22 tienen una probabilidad distinta de ser respondidos por los grupos G3 y G4, que se expresa en que los ítems 12 y 17 son más sensibles a mostrar la depresión en mujeres y los ítems 22, 28 y 30 en hombres. El funcionamiento diferencial de los ítems en los grupos de depresión respecto del género se encontró que los ítems 2 , $4,9,12,16,19,20,21,24,27,29,32,37$ y 38 se presentan más ajustados a la población masculina deprimida, pero las diferencias eran significativas en los ítems 12, 17, 28, 30 y 22.

\section{TABLA 2}

Análisis del funcionamiento diferencial de los items respecto del género en hombres deprimidos $(n=156)$ y mujeres deprimidas $(n=174)$

\begin{tabular}{|c|c|c|c|c|c|c|c|c|}
\hline \multirow{2}{*}{ Items } & \multirow{2}{*}{$\begin{array}{c}\text { Meassure } \\
\text { Hombres } \\
\text { Deprimidos }\end{array}$} & \multirow{2}{*}{$\begin{array}{c}\text { Meassure } \\
\text { Mujeres } \\
\text { Deprimidas }\end{array}$} & \multirow{2}{*}{$\begin{array}{l}\text { Diferencia } \\
\text { contraste }\end{array}$} & \multicolumn{3}{|c|}{ Probabilildad de Welkh } & \multicolumn{2}{|c|}{ Mantel Haenzel } \\
\hline & & & & $t$ & df & Prob & chi & Prob. \\
\hline 1 & -1.08 & -1.15 & 0.072 & 0.23 & 330 & 0.809 & 0.16 & 0.681 \\
\hline 2 & -0.6 & -0.3 & -0.3 & -1.1 & 330 & 0.268 & 1.6 & 0.203 \\
\hline 3 & -0.97 & -1.19 & 0.209 & 9.9 & 330 & 0.367 & 0.09 & 0.759 \\
\hline 4 & -0.09 & 0.29 & -0.387 & -1.58 & 330 & 0.11 & 1.96 & 0.157 \\
\hline 5 & -0.44 & -0.48 & 0.412 & 0.15 & 330 & 0.876 & 0.27 & 0.589 \\
\hline 6 & -0.85 & .081 & -0.03 & -0.12 & 330 & 0.9 & 1.86 & 0.171 \\
\hline 7 & -1.43 & -153 & 0.1 & 0.42 & 330 & 0.671 & 0.24 & 0.611 \\
\hline 8 & -0.75 & -0.94 & 0.186 & 0.7 & 330 & 0.483 & 0.36 & 0.542 \\
\hline 9 & -0.22 & 0.01 & -0.221 & -0.83 & 330 & 0.404 & 0.2 & 0.648 \\
\hline 10 & 0.29 & 0.74 & -0.443 & -1.74 & 330 & 0.077 & 3.33 & 0.061 \\
\hline 11 & 0.25 & -0.05 & 0.3 & 1 & 229 & 0.324 & 2.21 & 0.133 \\
\hline 12 & -1.19 & -0.56 & -0.618 & -2.59 & 330 & 0.012 & 3.29 & 0.06 \\
\hline 13 & 0.37 & 0.19 & 0.178 & 0.73 & 330 & 0.463 & 1.07 & 0.301 \\
\hline 14 & -0.45 & .0 .92 & 0.464 & 1.67 & 330 & 0.088 & 2.72 & 0.093 \\
\hline 15 & -1.65 & -1.42 & -0.231 & -0.79 & 330 & 0.434 & 0.04 & 0.832 \\
\hline 16 & 9.93 & 0.84 & 0.081 & 0.32 & 330 & 0.741 & 0.06 & 0.978 \\
\hline 17 & 0.84 & 0.32 & 0.524 & 2 & 229 & 0.043 & 2.28 & 0.132 \\
\hline 18 & -1.03 & -1.2 & 0.168 & 0.66 & 330 & 0.5 & 0.53 & 0.464 \\
\hline 19 & 1.17 & 1.42 & -0.243 & -0.87 & 330 & 0.379 & 2.27 & 0.132 \\
\hline 20 & 0.54 & 0.86 & -0.322 & -1.14 & 330 & 0.254 & 0.26 & 0.602 \\
\hline 21 & -0.41 & 0.11 & -0.531 & -1.87 & 330 & 0.061 & 2.01 & 0.152 \\
\hline 22 & 1.08 & 0.2 & 0.877 & 3.27 & 229 & 0.012 & 4.96 & 0.017 \\
\hline 23 & 0.67 & 0.53 & 0.133 & 0.55 & 330 & 0.582 & 0.03 & 0.083 \\
\hline 24 & -1.14 & -1.14 & 0.02 & 0.01 & 330 & 0.991 & 0.08 & 0.767 \\
\hline 25 & -0.68 & -0.33 & $\begin{array}{l}-0.3424 \\
-\end{array}$ & -1.36 & 330 & 0.173 & 0.09 & 0.749 \\
\hline 26 & -0.62 & -0.46 & -0.163 & -0.58 & 330 & 0.558 & 0.52 & 0.473 \\
\hline 27 & 0.2 & 0.14 & 0.062 & 0.23 & 330 & 0.812 & 0.16 & 0.681 \\
\hline 28 & -1.14 & -0.81 & 0.622 & 2.23 & 229 & 0.022 & 5.55 & 0.011 \\
\hline 29 & 0.39 & 0.16 & 0.221 & 0.86 & 330 & 0.3828 & 0.73 & 0.394 \\
\hline 30 & 1.05 & 0.27 & 0.766 & 2.88 & 229 & 0.041 & 3.03 & 0.083 \\
\hline 31 & -1.03 & -1.17 & 0.138 & 0.54 & 330 & 0.593 & 0.77 & 0.366 \\
\hline 32 & 1.01 & 09 & 0.113 & 0.42 & 330 & 0.67 & 1.14 & 0.278 \\
\hline 33 & 0.34 & 0.7 & -0.358 & -1.44 & 330 & 0.154 & 0.83 & 0.356 \\
\hline 34 & 0.76 & 0.65 & 0.102 & 0.4 & 330 & 0.688 & 0.57 & 0.443 \\
\hline 35 & 1.42 & 1.35 & 0.061 & 0.24 & 330 & 0.809 & 0.1 & 0.738 \\
\hline 36 & 0.89 & 1.01 & -0.111 & $\begin{array}{l}-0.44 \\
-0.43\end{array}$ & 330 & 0.608 & 1.05 & 0.302 \\
\hline 37 & -0.83 & -0.6 & -0.224 & -0.05 & 330 & 0.337 & 1.24 & 0.261 \\
\hline 38 & 0.88 & 1.27 & -0.383 & -1.37 & 330 & 0.166 & 1.56 & 0.208 \\
\hline 39 & 0.81 & 1.13 & -0.324 & -1.23 & 330 & 0.02207 & 2.82 & 0.087 \\
\hline 40 & 0.83 & 1.03 & -0.203 & -0.75 & 330 & 0.04521 & 0.03 & 0.954 \\
\hline \multicolumn{9}{|c|}{$\begin{array}{l}\text { Nota.MNSQ: Mínimos Cuadrados Normados; } \\
\text { RSME: Raíc Cuadrada del Error Cuadrático } \\
\text { Medio; Diferencia de las medidas: Diferencia } \\
\text { mesuarel; Diferencia del contraste: mayor a 0.5; } \\
\text { Índice de Rash Welch; Índice Manten Haenzel; } \\
\text { Prob: probabilidad esperada inferior a } 0.05 . \\
\text { Fuente: elaboración propia. }\end{array}$} \\
\hline
\end{tabular}

En general hombres y mujeres deprimidos expresaban algunos síntomas de depresión considerados masculinos, pero en todos los casos los puntajes obtenidos por los hombres eran notablemente mayores que los obtenidos por las mujeres. Excepto en la Ideación suicida medida a través del $\mathrm{CDH}$, en la que las mujeres mostraron una calificación media más alta, pero no en el Riesgo suicida evaluado desde el MINI en el que la media en los hombres fue más alta (Tabla 3). 
TABLA 3

Análisis comparativos calificación de los factores en grupos clínicos y no clínicos y entre hombres y mujeres deprimidos

\begin{tabular}{|c|c|c|c|c|c|c|c|c|}
\hline & \multicolumn{4}{|c|}{ No Clinicos $(n=310)$} & \multicolumn{4}{|c|}{ Cinicos $(n=330)$} \\
\hline & Min. & Máx & $x$ & $D E$ & Min & Máx & $x$ & $D E$ \\
\hline CDH Total & 0 & 120 & 52.21 & 16.06 & 42 & 133 & 71.33 & 22.88 \\
\hline Factor 1 . Auto oimagen negariva & 0 & 12 & 4.12 & 1.89 & 4 & 15 & 6.38 & 2.82 \\
\hline Factor 2 , Ideación suicida & 0 & 12 & 4.65 & 1.53 & 4 & 19 & 7.59 & 2.57 \\
\hline Factor 3. Pobre imagen social & 0 & 18 & 9.01 & 2.45 & 6 & 22 & 11.59 & 5.03 \\
\hline Factor 4. Afecto negaritio & 0 & 45 & 20.04 & 6.75 & 15 & 55 & 29.35 & 9.47 \\
\hline Factor 5. Desesperamza & 0 & 9 & 3.48 & 2.01 & 3 & 11 & 5.13 & 209 \\
\hline Factor 6. Evitación & 0 & 24 & 11.68 & 3.64 & 0 & 26 & 15.78 & 4.27 \\
\hline Entrevista MINI- Depresión & 0 & 0 & 0 & 0 & 2 & 31 & 6.39 & 3.5 \\
\hline Factor I: Severidad depresión actual & 0 & 0 & 0 & 0 & 2 & 12 & 4.06 & 287 \\
\hline Factor 2: Disstimia & 0 & 0 & 0 & 0 & 2 & 19 & 6.79 & 5.25 \\
\hline \multirow[t]{3}{*}{ Factor 4 : Riesgo snicida } & 0 & 0 & 0 & 0 & 0 & 5 & 3.22 & 1.71 \\
\hline & \multicolumn{4}{|c|}{ Mujeres Deprimidas $(n=174)$} & \multicolumn{4}{|c|}{ Hombres Deprimidos $(n=156)$} \\
\hline & Min. & Máx & $x$ & $D E$ & Min. & Máx. & $x$ & $D E$ \\
\hline CDH Total & 0 & 138 & 83.41 & 25.4 & 48 & 133 & 85.85 & 133 \\
\hline Factor 1. Autoimagen negativa & 0 & 15 & 1.09 & 1.61 & 4 & 15 & 1.14 & 1.68 \\
\hline Factor 2. Ideación suicida & 0 & 16 & 7.07 & 3.8 & 4 & 16 & 6.83 & 3.4 \\
\hline Factor 3. Pobre imagen social & 0 & 22 & 12.04 & 4.27 & 6 & 22 & 12.64 & 4.15 \\
\hline Factor 4 . Afecto negativo & 0 & 58 & 35.67 & 10.08 & 19 & 55 & 35.83 & 8.65 \\
\hline Factor 5. Desesperanza & 0 & 12 & 5.87 & 2.61 & 3 & 11 & 6.15 & 2.36 \\
\hline Factor 6 . Evitación & 0 & 26 & 15.13 & 4 & 9 & 26 & 16.71 & 3.92 \\
\hline Entrevista MNI - Depresiön & 5 & 31 & 15.64 & 7.7 & 5 & 31 & 18.53 & 8.15 \\
\hline Factor 1: Severidad depresion actual & 3 & 12 & 6.92 & 2.83 & 2 & 12 & 7.20 & 2.77 \\
\hline Factor 2: Distimia & 4 & 19 & 11.58 & 4.4 & 4 & 19 & 12.05 & 4.55 \\
\hline Factor 4: Riesso suicida & 0 & 5 & 1.09 & 1.61 & 0 & 5 & 2.98 & 1.68 \\
\hline
\end{tabular}

Nota.Min: Puntaje mínimo obtenido;

Max: Puntaje máximo obtenido; X: media; DE: Desviación estándar.

Fuente: elaboración propia.

Al comparar con el estadístico ANOVA, las medias de los puntajes obtenidos con el $\mathrm{CDH}$ y el los obtenidos con el MINI en hombres se encontró que no existían diferencias estadísticamente significativas, excepto en el factor Evitación que fue significativamente más alto con el $\mathrm{CDH}$ ( $p$ menor o igual a 0.05). Al comparar hombres y mujeres, los puntajes de los hombres tendían a ser mayores y presentaban menor dispersión en todos los casos (Tabla 4).
TABLA 4

Comparación de medias de Depresión y los factores de la Entrevista MINI y los del CDH

\begin{tabular}{lccc}
\hline \multicolumn{1}{c}{ Variable } & Media cuadrática & $\mathrm{F}$ & $p$ \\
\hline CDH Total & 197.21 & 0.28 & 0.501 \\
Factor 1. Autoimagen negativa & 0.66 & 0 & 0.939 \\
Factor 2. Ideación suicida & 6.93 & 0.74 & 0.65 \\
Factor 3. Empobrecimiento imagen social & 10.39 & 0.58 & 0.445 \\
Factor 4. Afecto negativo & 0.76 & 0 & 0.926 \\
Factor 5. Pesimismo & 2.28 & 0.39 & 0.548 \\
Factor 6. Evitación & 69.53 & 4.5 & 0.036 \\
& & & \\
Entrevista MINI - Depresión & 7.78 & 0.13 & 0.722 \\
Factor 1: Severidad depresión actual & 1.89 & 0.24 & 0.624 \\
Factor 2: Distimia & 6.06 & 0.24 & 0.584 \\
Factor 3: Riesgo suicida & 0.05 & 0.006 & 0.939 \\
\hline
\end{tabular}

Nota. p: Nivel de significancia menos o igual a 0.05; F: estadístico del ANOVA Fuente: elaboración propia.

Luego de identificar los ítems con funcionamiento diferencial, se hizo un paralelo entre los síntomas evaluados con estos ítems en el $\mathrm{CDH}$ y los criterios diagnósticos del DSM para episodio depresivo mayor y distimia. Se encontró que algunos síntomas de depresión en hombres son incluidos únicamente entre los criterios diagnósticos específicos de distimia (reducido autodominio, pesimismo, desesperanza, sentimientos de inadecuación y eficacia general disminuida), pero no para depresión mayor y otros trastornos del estado de ánimo. Otros síntomas como frustración e impaciencia no son considerados signos de depresión, aunque en los hombres deprimidos son evidentemente clínicos. Adicionalmente, en el DSM, solo se incluye ideación suicida y se evidencia los hombres frecuentemente reportan otras conductas autolesivas como abuso de drogas, promiscuidad y exposición voluntaria al riesgo (Tabla $5)$.

También en el DSM se incluye la tristeza como un criterio diagnóstico de depresión, aunque los hombres no expresan tristeza de forma directa, en su lugar reportan la sensación permanente de estar vacíos; así mismo, el aislamiento social y la disminución de la actividad y la productividad incluidos en el 
DSM paradójicamente en los hombres se presenta como incremento del intercambio social emocionalmente superficial, exceso de trabajo y actividad en general y realización de actividades que provocan alta excitación, agrupados en el $\mathrm{CDH}$ como incremento de conductas evitativas. En esta misma línea, en la distimia se describen síntomas como malestar clínicamente significativo o deterioro funcional general, aunque en hombres se registra como sensación de intranquilidad o ansiedad matutina. Finalmente, los cambios bruscos $y$ extremos en el comportamiento y los problemas en el intercambio social son síntomas identificados exclusivamente en hombres (Tabla 5).

\section{TABLA 5}

Paralelo entre sintomas de depresión en hombres incluidos en el CDH y los sintomas de depresión incluidos en el DSM-IV-y V $(2001,2013)$

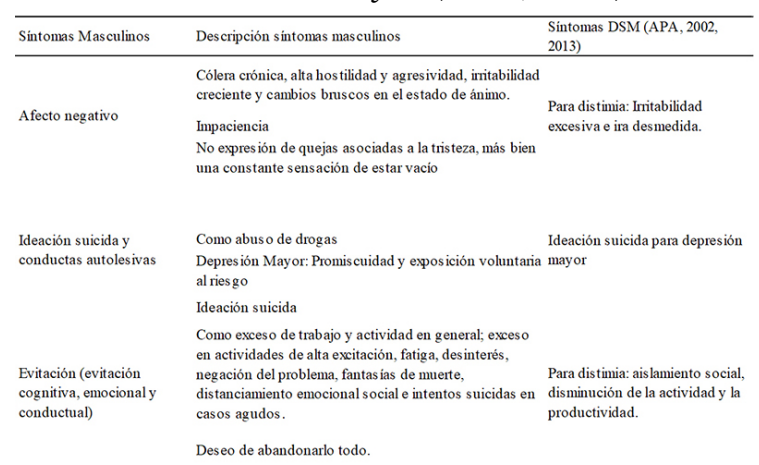

Fuente: elaboración propia.
TABLA 5 (Cont.)

Paralelo entre sintomas de depresión en hombres incluidos en el CDH y los síntomas de depresión incluidos en el DSM-IV- y V $(2001,2013)$

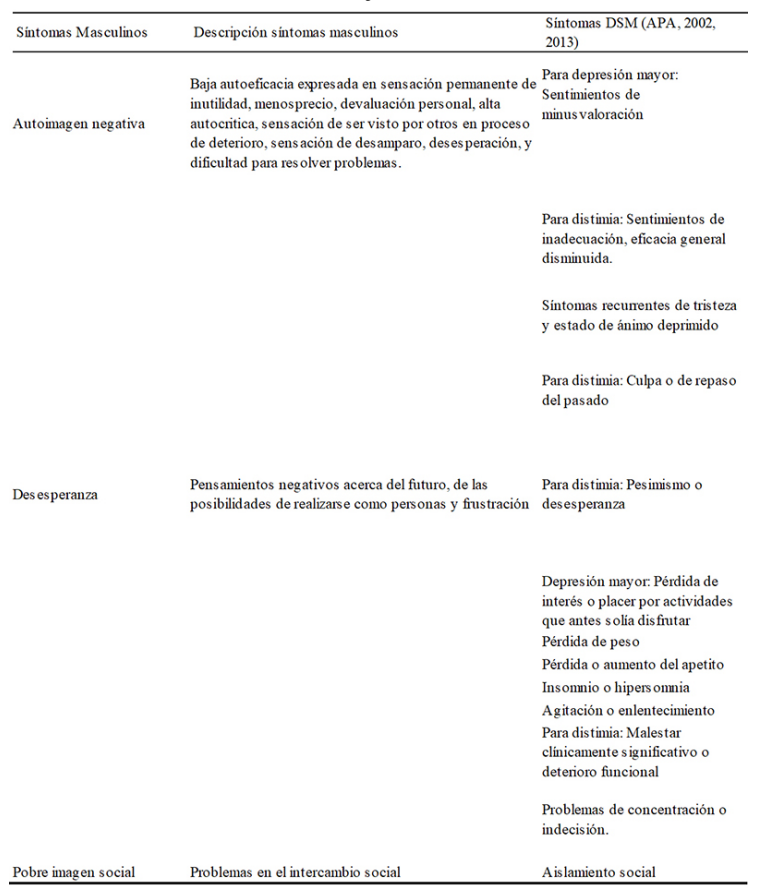

Fuente: elaboración propia.

\section{Discusión y conclusiones}

Es posible afirmar que existen síntomas específicos de depresión en hombres y que además se puede declarar sesgo diagnóstico en los criterios incluidos en el sistema DSM y CIE. En primer lugar, los ítems del $\mathrm{CDH}$ presentan el mejor ajuste a la población masculina, tienen funcionamiento diferencial por sexo por lo menos en el $40 \%$ de los ítems y los hombres tienden a presentar calificaciones más elevadas en los dos instrumentos usados para evaluar depresión en esta investigación.

La diferencia entre hombres y mujeres en la forma de responder al mismo cuestionario está asociada a la relevancia que los ítems tienen para cada población, es decir, que posiblemente para los hombres los síntomas evaluados en el $\mathrm{CDH}$ resultan más familiares y ajustados a su forma de actuar y pensar (deprimirse) que para el grupo de mujeres. Se puede pensar que cada uno de ellos mide aspectos diferentes y por ello se evocan 
procesamientos diversos en hombres y mujeres, es decir que miden dos dimensiones diferentes o no exactamente iguales en los dos grupos clínicos de estudio, y que probablemente las distribuciones multidimensionales no son exactas para cada grupo.

Tanto el funcionamiento diferencial de ítems como el mejor ajuste en la población de hombres indican que las diferencias encontradas no son debidas a un sesgo de medida de la prueba, sino a un sesgo real debido al impacto del sexo sobre la medida de la depresión; en otras palabras, el sesgo real identificado muestra que existen importantes diferencias cualitativas en el constructo entre los grupos medidos (Ackerman, 1992). Por tanto, se asume la diferencia sintomatológica entre hombres y mujeres y se acepta que hay varianza métrica, y se plantea que es necesario evaluar la depresión en los hombres con criterios específicos (Millsap \& Everson, 1993).

Es importante anotar que el $\mathrm{CDH}$ no puede ser usado para comparar hombres y mujeres, debido a que buena parte de los ítems tienen funcionamiento diferencial; así mismo, los resultados de estudios comparativos realizados con instrumentos que recogen los criterios diagnósticos del DSM deben ser tomadas con cautela y merecen reconfirmación en nuevas investigaciones.

Antes de ahondar en la discusión de los síntomas, es preciso anotar que en la misma línea de estudios transnacionales previos, los hombres expresaron cuadros de depresión más severos, con síntomas más agudos y estables, entre ellos evitación e ideación suicida (Lucht et al., 2003; Mittendorfer-Rutz et al., 2004; Van de Velde et al., 2010; Viana \& Andrade, 2012; Wenzel et al., 2005). Pero podría pensarse que los casos son más agudos porque los hombres acuden a los servicios sanitarios solo en casos de extrema urgencia (Álvarez \& Londoño, 2012), porque no reconocen fácilmente el estrés (Drevets \& Furey, 2009), o porque temen ser estigmatizados con un diagnóstico de enfermedad mental (Karasz, 2005).

La depresión en hombres entonces está caracterizada por síntomas como:
Autoimagen negativa: centrada principalmente en la percepción de baja autoeficacia que provoca autoevaluaciones de sí mismo basadas en las premisas de inutilidad y culpa, que ya habían sido documentadas como síntoma de depresión en hombres por varios autores (Álvarez \& Londoño, 2012; Kilmartin, 2005; Lehti \& Johansson, 2010; Strömberg et al., 2010).

La pobre valoración de la capacidad personal combinada con alta exigencia personal (p. ej., Franchi, 2010; Scher, Ingram, \& Segal, 2005) es fuente de permanentes censuras, excesiva autocrítica y de la sensación incapacidad de afrontar incluso las situaciones cotidianas, que los llevan a sentirse frustrados e inútiles. Se ha planteado que la pobre expectativa de autoeficacia proviene de estándares estrictos y ambiciosos de desempeño, difíciles de cumplir, que le provocan sentimientos de inferioridad y culpabilidad (González, Ibáñez, Rovella, López, \& Padilla, 2013; Stoeber \& Otto, 2006).

En estudios previos, se había confirmado que los hombres manejan más autoesquemas de autonomía que las mujeres, tienen necesidad permanente de independencia, privacidad, autodeterminación y libertad (Hankin, 2010; Liu \& Alloy, 2010). Al interpretar los estándares sociales, los hombres deprimidos esperan ser y actuar de forma perfecta e independiente y libre (Mallinger, 2009; Zapata \& Giner, 2009), ya que creen que pueden y deben autodeterminar totalmente sus extremas metas personales (Jahromi et al., 2012), y no cumplirlas les genera autocrítica hostil (hosein ghahramani, Ali Besharat, \& naghipour, 2011) y sensación de culpa.

El resultado entonces es una valoración selectiva negativa de sí mismo asociado a estados depresivos de alta severidad (Iacoviello, Alloy, Abramson, Whitehouse, \& Hogan, 2006; Zapata $\&$ Giner, 2009), al tiempo que acrecientan el miedo de recibir rechazo, desaprobación o critica de quienes los rodean (van Tuijl, de Jong, Sportel, de Hullu, \& Nauta, 2013; Weeks, Jakatdar, \& Heimberg, 2010; Zeigler-Hill, 2011).

Desesperanza o visión pesimista del futuro: expresada como preocupación por la falta de oportunidades futuras para realizarse como 
persona y por la expectativa de que no se lograrán la metas establecidas (Mahoney, McEvoy, \& Moulds, 2012; McLaughlin \& Nolen-Hoeksema, 2011), debido a que considera que no cuenta con los recursos personales para hacerles frente. Para varios autores, este pesimismo es una forma de expresar la tendencia a la preocupación e intolerancia a la incertidumbre, pues de forma permanente se anticipa un futuro negativo, y la sensación de estar vacío aparece porque la persona no sabe qué ocurrirá y tampoco se siente preparada para responder ante las demandas ambientales y sociales (Fergus \& Wu, 2011; González et al., 2013; Rosellini Fairholme, \& Brown, 2011; Rovella, González, Ibáñez, \& Peñate, 2011).

Empobrecimiento de la imagen social: expresada en la preocupaban excesiva por la imagen social reflejada (Joiner, 2003; Wang et al., 2012) y por el deterioro de la misma. Este temor al deterioro de la imagen social podría originarse en la pobre percepción que tienen de sí mismos y de sus propias capacidades; temor que los predispone a detectar más fácilmente información social negativa (Gollan, Pane, McCloskey, \& Coccaro, 2008; Gotlib \& Joormann, 2010; Alter, 2011; Lester, Mathews, Davison, Burgess, \& Yiend, 2011; Sánchez \& Vázquez, 2012). Por el contrario, las mujeres deprimidas mostraron menor preocupación al respecto, porque socialmente se les permite la solicitud de soporte social.

Esta incrementada sensibilidad social, ya asociada a depresión por otros autores (Wilhem, Boyce, \& Brownhil, 2004), ha sido definida por Ellis y Rutherford (2008) como perfeccionismo social impuesto por la cultura que es trasmitido de forma directa con mandatos expresados a través de prácticas culturales de competitividad, funcionalidad y fuerza. Precisamente, este tipo de perfeccionismo centrado en la valoración hecha por otros, ha sido ligado a depresiones más severas y conducta suicida (Egan, Wade, \& Shafran, 2011; Franchi, 2010; O'Connor et al., 2012; Rasmussen, Elliot, \& O'Connor, 2012).

Sensación permanente de estar vacío: descrita como la impresión de que hace falta algo dentro, basada en la falta de reevaluación adaptativa de los eventos que causan tristeza y de la respuesta especifica ante la emoción (Bonanno \& Kaltman, 2001; Instituto Nacional de Salud Mental de Estados Unidos [NIHM], 2005); ambas necesarias para que se reconozca efectivamente el reajuste comportamental (Clore \& Colcombe, 2003; Niemeyer, 2006; Welling, 2003), o al menos para diferenciar la sensación de tristeza de la de vacío.

Aquí surge un problema crucial: si la base emocional del trastorno depresivo es la persistencia de la tristeza (Bonanno, Goorin, \& Coifman, 2008; Viana \& Andrade, 2012), de qué manera se garantiza que los síntomas masculinos descritos son debidos a la presencia del trastorno. La respuesta reside en el uso de diversas medidas clínicas de reconfirmación que señalaron que los hombres que hicieron parte de la población clínica padecían una depresión, pero es necesario comprobar esta hipótesis con nuevas investigaciones.

En Occidente, se enseña desde la infancia a los hombres a evitar la tristeza, de tal modo que se desconoce su valor y se limita la posibilidad de aprender a afrontar de forma adaptativa futuras pérdidas. De hecho, en las sociedades machistas, los hombres que lloran o se deprimen son vistos como poco masculinos (Adinkrah, 2012) y en general no esperan obtener apoyo porque eso implica asumir socialmente la incapacidad personal o la culpa.

Incremento en la actividad laboral, social y sexual: los hombres no muestran disminución en la actividad en general, como ocurre en las mujeres, por el contrario, se presenta trabajo excesivo, mayor intercambio social emocionalmente superficial y cambio frecuente de parejas sexuales, como indicios de que el afecto positivo no es necesariamente bajo entre los hombres deprimidos como lo plantearon previamente Anderson y Hope (2008) y Echeburúa (2012). Es decir, que es posible que existan importantes diferencias en la expresión del afecto entre hombres y mujeres deprimidos, variaciones que determinan los síntomas, la severidad del trastorno y, por tanto, el tipo de intervenciones efectivas. 
Para diversos autores, los hombres con depresión cambian frecuentemente de pareja $\mathrm{y}$ son promiscuos debido a que buscan experimentar nuevas sensaciones (Álvarez \& Londoño, 2011; Fuss et al., 2010; McCusker $\&$ Galupo, 2011) y pretenden demostrar que conservan la capacidad sexual y el arrojo necesario para involucrarse en situaciones prohibidas (Broom \& Tovey, 2009; Kilmartin, 2005; Watkins, 2011).

Evitación: La tendencia a responder de forma evitativa, que caracteriza a los varones deprimidos, ya había sido descrita en estudios previos (Álvarez \& Londoño, 2011; Kilmartin, 2005; Lehti \& Jonanson, 2010; Marmorstein \& Iacono, 2011; Simon \& Nath, 2004). Para Bennett et al. (2005) esta disposición evitativa tiene dos objetivos: uno cognitivo dirigido a evadir la preocupación acerca del futuro y el otro emocional encaminado a minimizar la sensación de vacío que les provoca la depresión.

De este modo el consumo de drogas (alcohol y energizantes) es un mecanismo usado para minimizar las sensaciones negativas asociadas a la depresión o al agotamiento provocado por el exceso de trabajo (Cote-Menéndez, Rangel-Garzón, Sánchez-Torres, \& MedinaLamus, 2011; Martins \& Goerlick, 2011). Es importante anotar que el consumo de alcohol y otras drogas puede tener efectos potenciadores de la severidad del trastorno depresivo, además de empobrecer los resultados terapéuticos e incrementar el riesgo suicida (Glasner-Edwards et al., 2008; Worley et al., 2012).

Conductas autolesivas y tendencia suicida: En la muestra objeto de estudio se encontró que en general los hombres deprimidos presentan mayor frecuencia de conductas autolesivas y riesgo suicida, especialmente cuando la depresión se hace más severa. En esta misma línea, otros autores ya habían relacionado con depresión mayor, conductas autolesivas como la ideación suicida (O'Connor et al., 2012; Rasmussen et al., 2012), los intentos de suicidio (Egan et al., 2011; Franchi, 2010) y la suicidios efectivos usando métodos más agresivos (Kilmartin, 2005).

Entre las conductas autolesivas reportadas, están la exposición voluntaria al riesgo (realización de deportes extremos sin usar protección), el abuso en el consumo de drogas, conducir ebrio y tener relaciones sexuales sin protección. Este tipo de conductas, consideradas por algunos como para suicidas, no son más que una expresión de ira hacia sí mismos, pueden ser realmente extremas y terminar en un suicidio (Arsenault-Lapierre, Kim, \& Turecki, 2004).

Comportamiento inusual: Hace referencia a la presencia de comportamientos que se encontraban fuera del espectro conductual característico de las personas antes de haberse deprimido. Síntoma previamente registrado en un estudio con población proveniente de países industrializados Kilmartin (2005) y en población colombiana (Álvarez \& Londoño, 2011). Para Kilmartin (2005) y Bowen, Wang, Balbuena, Houmphan y Baetz (2013) estas fluctuaciones ocurren debido a la inestabilidad emocional frecuente en hombres con depresión, pero que ha sido poco estudiada porque no es un síntoma registrado en los instrumentos de evaluación disponibles (Mazer \& Juruena, 2013). Es posible pensar que los cambios de comportamiento y las fluctuaciones del estado de ánimo hacen que los hombres perciban como incontrolable su situación y refuercen esquemas de incapacidad y bajo control emocional.

De otra parte, $y$ al igual que en estudios previos realizados en población colombiana (Álvarez \& Londoño, 2011; Londoño \& González, 2016) y europea (Möller-Leimkühler \& Yücel, 2010), mujeres con alta masculinidad presentaron algunos de los síntomas considerados propios de los hombres. Esto conduce a pensar que las diferencias en los síntomas no son debidas al sexo sino al género, es decir que la expresión de la masculinidad/feminidad que cambian a través del tiempo, debido al impacto de transformaciones económicas, políticas y sociales que tienen impacto en la cultura y en los roles que deben asumir hombres y mujeres.

También pudo establecerse que existe un grupo de síntomas de depresión que parecen ser independientes del género y únicamente debidos al estado depresivo; para Peñate, Bello, García, Rovella y Pino-Sedeño (2014) estos son síntomas centrales de depresión (afecto triste, anhedonia y 
baja autoestima) y cuya identificación facilitaría la diferenciación de los trastornos de alta comorbilidad, como la depresión y la ansiedad, entre otros. Pero los hallazgos en este estudio apenas permiten evidenciar la importancia de trabajar en el tema.

Los resultados del presente estudio han hecho evidente que los sistemas nosológicos, conservado casi en su totalidad en la quinta versión y CIE-10 (OPS, 2010), actualmente usados por psiquiatras y psicólogos en el diagnóstico de depresión, no reconocen las diferencias de género aquí evidenciadas. Entonces, todo apunta a que existen síntomas específicos de depresión masculina, que estos no se incluyen en el sistema nosológico DSMIV-TR y DSM V (APA, 2002, 2013) y CIE-10 (Organización Panamericana de Salud [OPS], 2010) y que los criterios diagnósticos pueden ser ajustados para mejorar su alcance en la detección y diagnóstico de depresión en los hombres. Aunque aún es preciso conducir estudios transculturales que permitan contrastar si los resultados obtenidos en este estudio son generalizables a otros grupos poblacionales.

\section{Referencias}

Ackerman, T. A. (1992). A didactic explanation of item bias, item impact, and item validity from a multidimensional perspective. Journal of Educational Measurement, 29, 67-91. https://doi.org/10.1111/ j.1745-3984.1992.tb00368.x

Adinkrah, M. (2012). Better dead tan dishonored: Masculinity and male suicidal behavior in contemporary Ghana. Social Science EO Medecine, 74(4), 474-481. https:// doi.org/10.1016/j.socssimed.2010.10.011

Alter, K. (2011). 'Emotion'. 'The essence of Human communication - The processing of emotional information'. Comment on: Brück C., Kreifelts B., Wildgruber D. Emotional voices in context: A neurobiological model of multimodal affective information processing. Physics of Life Reviews, 8(4), 406-407. https:// doi.org/10.1016/j.plrev.2011.10.016

Álvarez, N., \& Londoño, C. (2011). ¿Se deprimen los hombres? iDiferente de las mujeres? Cuestionario de Depresión en Hombres. Madrid: Editorial Académica Española.

Álvarez, N., \& Londoño, C. (2012). Diseño y validación del Cuestionario de Depresión en Hombres (Tesis de maestría inédita). Universidad Católica de Colombia, Bogotá.

American Psycological Asociation. (2013). Diagnostic and statistical manual of mental disorders, DSM-V. Washington, DC: American Psychiatric Association

American Psychiatric Association. (2002). Diagnostic and statistical manual of mental disorders DSM-IV TR. Washington, DC: Masson.

Anderson, E., \& Hope, D. A. (2008). A review of the tripartite model for understanding the link between anxiety and depression in youth. Clinical Psychology Review, 28, 275-287. https:// doi.org/10.1016/j.cpr.2007.05.004

Angst, J., Gastpar, M., Lépine, J. P., Mendlewicz, J., \& Tylee, A. (2002). Gender differences in depression. Epidemiological findings from the European Depression, I and II studies. European Archives of Psychiatry and Clinical Neuroscience, 252, 201-209. https:// doi.org/10.1007/s00406-002-0381-6

Arsenault-Lapierre, G., Kim, C., \& Turecki, G. (2004). Psychiatric diagnoses in 3275 suicides: A meta-analysis. BCM Psychiatry, 4, 37-47. https:// doi.org/10.1016/j.jmhg.2005.05.004

Bech, P., Olsen, L. R., Kjoller, M., \& Ramussen, N. K. (2003). Measuring well-being rather than the absence of distress symptoms: A comparison of the SF-36 Mental Health Subscale and the WHO-Five Well-Being Scale. International Journal of Methods in Psychiatric Research, 12(2), 85-91. https:// doi.org/10.1002/mpr.145

Bennett, D., Ambrosini, P., Kudes, D., Metz, C., \& Rabinovic, H. (2005). Gender differences in adolescent depression: Do symptoms differ for boys and girls? Journal of 
Affective Disorders, 89(1-3), 35-44. https:// doi.org/10.1016/j.jad.2005.05.020

Beutel, M., Glaesmer, H., Wiltink, J., Marian, H., \& Brahler, E. (2010). Life satisfaction, anxiety, depression and resilience across the life span of men. The Aging Male, 13, 32-39. https://doi.org/10.1186/cc9210

Bonanno, G., \& Kaltman, S. (2001). The varieties of grief experience. Clinical Psychology Review, 21, 705-734. https:// doi.org/10.1016/S0272-7358(00)00062-3

Bonnano, G., Goorin, L., y Coifman, K. (2008). Sadness and Grieff. En Lewis, M., Haviland-Jones, J. y J. Feldman (2008). Handbook of Emotions. (3rd). (1-21). NY USA: Guildford Press.

Bowen, R., Wang, Y., Balbuena, L., Houmphan, A., \& Baetz, M. (2013). The relationship between mood instability and depression implications for studying and treating depression. Medical Hypotheses, 81 (3), 459-462. https:// doi.org/0306987713002880

Branney, P., \& White, A. (2008). Big boys don't cry: Depression and men. Advances in Psychiatric Treatment, 14, 256-262. https:// doi.org/10.1192/apt.bp.106.003467

Broom, A., \& Tovey, P. (2009). Men's health: Body, identity and social context. Londres: Wiley.

Brownhill, S., Wilhelm, K., Barclay, L., \& Schmied, V. (2005). Big build: Hidden depression in men. Australian and New Zeland Journal of Psychiatry, 39, 921-931. https://doi.org/10.1111/ j.1440-1614.2005.01665.x

Carvalho, J., \& Hopko, D. (2011). Behavioral theory of depression: Reinforcement as a mediating variable between avoidance and depression. Journal of Behavior Therapy and Experimental Psychiatry, 42 (2), 154-162. https://doi.org/10.1016/j.jbtep.2010.10.001

Clore, G., \& Colcombe, S. (2003). The parallel worlds of affective concepts and feelings. En J. Musch \& K. C. Klauer (Eds.), The psychology of evaluation: Affective processes in cognition emotion (pp. 335-369). Mahwah, NJ: Erlbaum.
Cochran, S. V., \& Rabinowitz, F. E. (2003). Gender-sensitive recommendation for assessment and treatment of men. Professional Psychology: Research and Practice, 34, 132-140. https:// doi.org/10.1037/0735-7028.34.2.132

Cote-Menéndez, M., Rangel-Garzón, C. X., Sánchez-Torres, M. Y., \& MedinaLamus, A. (2011). Bebidas energizantes: iHidratantes o estimulantes? Revista Facultad de Medicina Universidad Nacional de Colombia, 59(3), 255-266. Recuperado de http://www.revistas.unal.edu.co/index.p hp/revfacmed/article/view/26461/38956

Drevets, W. C., \& Furey, M. L. (2009). Depression and the brain. En R. S. Larry (Ed.), Encyclopedia of neuroscience (pp. 459-470). Oxford: Academic Press. https://doi.org/10.1016/ B978-008045046-9.00846-9

Echeburúa, E. (2012). ¿Existe realmente la adicción al sexo? Adicciones, 24(4), 281-286. Recuperado de http://www.redaly c.org/pdf/2891/289124639001.pdf

Egan, S., Wade, T., \& Shafran, R. (2011). Perfeccionism as a transdiagnostic process: A clinical review. Clinical Psycology Review, 31, 203-212. https:// doi.org/10.1016/j.cpr.2010.04.009

Ellis, T. E., \& Rutherford, B. (2008). Cognition and suicide: Two decades of progress. International Journal of Cognition Therapy, 1(1), 47-68. https:// doi.org/10.1521/ijct.2008.1.1.47

Emslie, C., Ridge, D., Ziebland, S., \& Hunt, K. (2006). Men's account of depression: Reconstructing or resisting hegemonic masculinity? Social Science 83 Medicine, 62, 2246-2257. https://doi.org/10.1016/ j.socscimed.2009.01.004

Essau, C., Lewinsohn, P., Seeley, J., \& Sasagawa, S. (2010). Gender differences in the developmental course of depression. Journal of Affective Disorders, 127(1-3), 185-190. https://doi.org/10.1016/j.jad.2010.05.016

Fava, M., Nolan, S., Kradin, R., \& Rosenbaum, J. (1995). Gender differences in hostility among depressed and medical outpatients. 
The Journal of Nervous and Mental Disease, 18, 10-14. https://doi.org/10.1007/ s10802-010-9458-2

Franchi, S. (2001). La depresión en el hombre. Interdisciplinaria, 18(2), 135-154. Recuperado de http://www.redalyc.org/arti culo.oa?id=18011325002

Franchi, S. (2010). Estilo de personalidad perfeccionista y depresión. Perspectivas en Psicología, 13(15), 53-64. Recuperado de http://www.umanizales.edu.co/publicaci ones/campos/sociales/...revista13/.pdf

Fergus, T., \& Wu, K. D. (2011). Searching for specificity between cognitive vulnerabilities and mood and anxiety symptoms. Journal of Psychopathology and Behavioral Assessment, 33, 446-458. https:// doi.org/10.1007/s10862-011

Fuss, J., Ben Abdallah, N., Vogt, M., Touma, C., Pacifici, P., \& Palme, R. (2010). Voluntary exercise induces anxiety-like behavior in adult C57BL/6J mice correlating with hippocampal neurogenesis. Hippocampus, 20(3), 364-376. https://doi.org/10.1002/ hipo2.0634

Galea, S., Ahern, J., Nandi, E., Tracy, M., Beard, J., \& Vlavov, D. (2007). Urban neighborhood poverty and the incidence of depression in a populationbased cohort study. Annals of Epidemiology, 17(3), 171-179. https://doi.org/10.1016/ j.annepidem.2006.07.008

Gollan, J. K., Pane, H. T., McCloskey, M. S., \& Coccaro, E. F. (2008). Identifying differences in biased affective information processing in major depression. Psychiatry Research, 59, 18-24. https:// doi.org/10.1016/j.psychres.2007.06.011

González, M., Ibáñez, I., Rovella, A., López, M., \& Padilla, L. (2013). Perfeccionismo e intolerancia a la incertidumbre: relaciones con variables psicopatológicas. International Journal of Clinical and Health Psychology, 21(1), 81-101. https://doi.org/10.1016/ j.brat.2009.11.013

Gotlib, I., \& Joormann, J. (2010). Cognition and depression: Current status and future directions. Annual
Review of Clinical Psychology, 6, 285-312. https://doi.org/10.1146/ annurev.clinpsy.121208.131305

Haeffel, G., Abramson, L., Brazy, P., Shah, J., Teachman, B., \& Nosek, B. (2007). Explicit and implicit cognition: A preliminary test of a dual-process theory of cognitive vulnerability to depression. Behaviour Research and Therapy, 45(6), 1155-1167. https://doi.org/10.1016/j.brat.2006.09.003

Hankin, B. L. (2010). Personality and depressive symptoms: Stress generation and cognitive vulnerabilities to depression in a prospective daily diary study. Journal of Social and Clinical Psychology, 29(4), 369-401. https://doi.org/10.1521/ jscp.2010.29.4.369

Hölzel, L., Härter, M., Reese, C., \& Kriston, L. (2011). Risk factors for chronic depression: A systematic review. Journal of Affective Disorders, 129, 1-13. doi: 10.1016/ j.jad.2010.03.025

hosein ghahramani, M., Ali Besharat, M., \& naghipour, B. (2011). An examination of the relationship between perfectionism and self-esteem in a sample of student athletes. Procedia-Social and Behavioral Sciences, 30, 1265-1271. https://doi.org/10.1016/ j.sbspro.2011.10.245

Iacoviello, B., Alloy, L., Abramson, L., Whitehouse, W., \& Hogan, W. (2006). The course of depression in individuals at high and low cognitive risk for depression: A prospective study. Journal of Affective Disorders, 93(1-3), 61-69. https:// doi.org/10.1016/j.jad.2006.02.012

Instituto Nacional de Medicina Legal y Ciencias Forenses. (2011). Forensis 2011: datos para la vida (Informe para la prensa). Bogotá: Autor. Recuperado de http://www.medicinalegal.gov.co/index. php? option $=$ comwrapperyItemid $=323$

Jahromi, F. G., Naziri, G., \& Barzegar, M. (2012). The relationship between socially prescribed perfectionism and depression: The mediating role of maladaptive cognitive schemas. Procedia-Social and 
Behavioral Sciences, 32, 141-147. https:// doi.org/10.1016/j.sbspro.2012.01.023

Joiner, T. (2003). "Contagion" of suicidal symptoms as a function of assortative relating and shared relationship stress in college roommates. Journal of Adolescence, 26, 495-504. https://doi.org/10.1016/ S0140-1971(02)00133-1

Juárez, F., \& Guerra, R. (2011). Características socioeconómicas y salud en personas pobres y desplazadas. Psicologia: Teoria e Pesquisa, 27(4), 511-519.

Karasz, A. (2005). Cultural differences in conceptual models of depression. Social Science $\mathcal{B}$ Medecine, 60 (7), 1625-1636. https://doi.org/10.1016/ j.socscimed.2004.08.011

Katz, M., Wetzler, S., \& Cloitre, M. (1993). Expressive characteristics of anxiety in depressed men and women. Journal of Affective Disorders, 28, 267-277. https:// doi.org/10.1192/apt.bp.106.003467

Kilmartin, C. (2005). Depression in men: Communication, diagnosis and therapy. Journal of Men's Health and Gender, 2(1), 95-99. https:// doi.org/10.1080/17482620701547008

Londoño, C., \& González, M. (2016). Prevalencia de depresión y factores asociados en hombres. Acta Colombiana de Psicología, 19(2), 315-329. Recuperado de http://www.scielo.org.co/pdf/acp/v19n2/ es_v19n2a13.pdf

Londoño, C., Peñate, W., \& González, M. (2016). Validación diferencial y discriminante del Cuestionario de Depresión para Hombres. Terapia Psicológica, 34(2), 129-142. https://doi.org/10.4067/ S0718-48082016000200005

Lehti, A., \& Johansson, E. (2010). The Western Gaze: An analysis of medical research publications concerning the expressions of depression, focusing on ethnicity and gender. Health Care for Women International, 31, 100-112. https:// doi.org/10.1080/07399330903067861

Lester, K. J., Mathews, A., Davison, P. S., Burgess, J. L., \& Yiend, J. (2011). Modifying cognitive errors promotes cognitive wellbeing: A new approach to bias modification. Journal of Behaviour Therapy and Experimental Psychiatry, 42, 298-308. https://doi.org/10.1016/j.jbtep.2011.01.001 Levola, J., Holopainen, A., \& Aalto, M. (2010). Depression and heavy drinking occasions: A cross-sectional general population study. Addictive Behavior, 36(4), 375-380.

Liu, R. T., \& Alloy, L. B. (2010). Stress generation in depression: A systematic review of the empirical literature and recommendations for future study. Clinical Psychology Review, 30, 582-593. https:// doi.org/10.1016/j.cpr.2010.04.010

Lucht, M., Schaub, R. T., Meyer, C., Hapke, U., Rumpf, H. J., Bartels, T., ... Joh, U. (2003). Gender differences in unipolar depression: A general population survey of adults between age 18 to 64 of German nationality. Journal of Affective Disorders, 77(3), 203-211. https://doi.org/10.1016/ S0165-0327(02)00121-0

McCusker, M., \& Galupo, M. (2011). The impact of men seeking help for depression on perceptions of masculine and feminine characteristics. Psychology of Men $\mathcal{E}$ Masculinity, 12 (3), 275-284. https:// doi.org/10.1037/a0021071

McLaughlin, K., \& Nolen-Hoeksema, S. (2011). Rumiation as a transdiagnostic factor in depression and anxiety. Behavior Research and Therapy, 49(3), 186-193. https:// doi.org/10.1016/j.brat.2010.12.006

Mahoney, A., McEvoy, P., \& Moulds, M. (2012). Psychometric properties of the Repetitive Thinking Questionnaire in a clinical sample. Journal of Anxiety Disorders, 26, 359-367. https:// doi.org/10.1016/j.janxdis.2011.12.003

Mallinger, A. (2009). The myth of perfection: Perfectionism in the obsessive personality. American Journal of Psychotherapy, 63(2), 103-131.

Marmorstein, N. R., \& Iacono, W. G. (2011). Explaining associations between cannabis use disorders in adolescence and later major depression: A test 
of the psychosocial failure model. Addictive Behaviors, 36, 773-776. https:// doi.org/10.1016/j.addbeh.2011.02.006

Martins, S. S., \& Gorelick, D. A. (2011). Conditional substance abuse and dependence by diagnosis of mood or anxiety disorder or schizophrenia in the U.S. population. Drug Alcohol Dependency, 119, 28-36. https:// doi.org/10.1016/j.drugalcdep.2011.05.010

Matud-Aznar, M. (2008). Género y salud. Suma Psicológica, 15(1), 75-94.

Mazer, A. K., \& Juruena, M. (2013). Differential diagnosis between bipolar disorder and borderline personality disorder based on early life stress and psychoneuroendocrine assessment. European Psychiatry, 28(1), 122-134. https://doi.org/10.1016/ S0924-9338(13)77077-9

Merikangas, K. R., Jin, R., He, J. -P., Kessler, R., Lee, S., Sampson, N., ... Zarkov, Z. (2011). Prevalence and correlates of bipolar spectrum disorder in the World Mental Health Survey Initiative. Archive General of Psychiatry, 68(3), 241-251. https:// doi.org/10.1001/archgenpsychiatry.2011.12

Millsap, R. E., \& Everson, H. T. (1993). Methodology review: Statistical approaches for assessing measurement bias. Applied Psychological Measurement, 17(4), 297-334. doi: 10.1177/014662169301700401

Mittendorfer-Rutz, W. E. (2006). Trends of youth suicide in Europe during the 1980 `s and 1990`s. Gender Differences and Implications for Prevention, 3, 250-257. https://doi.org/10.1016/j.jmhg.2006.02.006

Mittendorfer-Rutz, W. E., Rasmussen, F., \& Wasserman, D. (2004). Restricted fetal growth and adverse maternal psychosocial and socioeconomic conditions as risk factors for suicidal behaviour of offspring: A cohort study. Lancet, 364(9440), 1135-1140. doi: 10.1016/ S0140-6736(04)17099-2

Möller, A. M., Heller, J., \& Paulus, N. C. (2006). Subjective well-being and 'male depression' in male adolescents.
Journal Affects Disorders, 7, 65-72. https:// doi.org/10.1016/j.jad.2006.07.007

Möller-Leimkühler, A. M. (2002). Barriers to help-seeking by men: A review of socio-cultural and clinical literature with particular reference to depression. Journal of Affective Disorders, 71, 1-9. https:// doi.org/10.1016/S0165-0327(01)00379-2

Möller-Leimkühler, A. M., \& Yücel, M. (2010). Male depression in females? Journal of Affective Disorders, 121, 22-29. https:// doi.org/10.1016/j.jad.2009.05.007

Moore, S. M., \& Hadjiyannakis, K. (2002). The social enviroment and depression: Focusing on severe life stress. En I. Gotlib \& C. Hammen (Eds.), Handbook of depression (pp. 314-340). Nueva York: Guilford.

Mossakowski, K. N. (2008). Dissecting the influence of race, ethnicity, and socioeconomic status on mental health in young adulthood. Research on Aging, 30(6), 649-671. https:// doi.org/10.1177/0164027508322693

National Institute of Mental Health. (2005). Men and depression (NIH Publication No. QF 11-5300). Bethesda, Maryland: Autor. Recuperado de http://www.nimh.nih.gov/health/public ations/men-and-depression/index.shtml

Niemeyer, R. A. (2006). Widowhood, grief and the quest for meaning: A narrative perspective on resilience. En D. Carr, R. M. Nesse \& C. B. Wortman (Eds.), Spousal bereavement in late life (pp. 227-252). Nueva York: Springer.

O'Connor, S., Jobes, D., Comtois, K., Atkins, D., Janis, K., Chessen, C., \& Landes, S. (2012). Ideación suicida: Identifying outpatients with entrenched suicidal ideation. Suicide and Life-Threatening Behavior, 42 (2), 173-184. https://doi.org/10.1111/ j.1943-278X.2012.00080.x

Ordaz, S., \& Luna, B. (2012). Sex differences in physiological reactivity to acute psychosocial stress in adolescence. Psychoneuroendocrinology, 37(8), 1135-1157. https://doi.org/10.1016/ j.psyneuen.2012.01.002 
Organización Mundial de la Salud. (2009). Gender and mental health. Ginebra: Autor. Recuperado de http://www. Who.int/gende $\mathrm{r} /$ henderandhealth.html

Organización Panamericana de la Salud. (2012). Día Mundial de la Salud Mental: la depresión es el trastorno mental más frecuente. Buenos Aires: Autor. Recuperado de http://www.pa ho.org/arg/index.php?option=com_conten $\mathrm{t} \&$ view $=$ article\&id $=1047 \&$ Itemid $=325$

Organización Panamericana de Salud. (2010). Sistema de Clasificación Estadística Internacional de Enfermedades y Problemas Relacionados con la Salud. Recuperado de http://www.paho.org/hq/index.php?opti on $=$ com content\&view $=$ article $\& i d=539$ $5 \&$ Itemid $=2560$ \&lang $=\mathrm{es}$

Palomar, J., \& Cienfuegos, Y. (2007). Pobreza y apoyo social: un estudio comparativo en tres niveles socioeconómicos. Revista Interamericana de Psicología, 41 (2), 177-188. Recuperado de http://www.revista.unam.m x/vol.6/.../nov_art111.pdf

Peñate, W., Bello, R., García, A., Rovella, A., \& Pino-Sedeño, T. (2014). Características psicométricas del Cuestionario Básico de Depresión en una muestra de adolescentes. Anales de Psicología, 30(1), 143-149. https:// doi.org/10.6018/analesps.30.1.185311

Rasmussen, S., Elliot, M., \& $\mathrm{O}^{\prime}$ Connor, R. C. (2012). Psychological distress and perfectionism in recent suicide attempters: The role of behavioural inhibition and activation. Personality and Individual Differences, 52(6), 680-685. https://doi.org/10.1016/j.paid.2011.12.011

Rihmer, Z., Rutz, W., \& Pihlgren, H. (1995). Depression and suicide on Gotland: An intensive study of all suicides before and after a depression-training programme for general practitioners. Journal of Affective Disorders, 35, 147-152. https:// doi.org/10.1016/0165-0327(95)00055

Rosellini, A., Fairholme, C., \& Brown, T. (2011). The temporal course of anxiety sensitivity in outpatients with anxiety and mood disorders: Relationships with behavioral inhibition and depression. Journal of
Anxiety Disorders, 25(4), 615-621. https:// doi.org/10.1016/j.janxdis.2011.02.001

Rovella, A., González, M., Ibáñez, I., \& Peñate, W. (2011). Preocupación-rasgo y trastorno de ansiedad generalizada en una muestra de la población general: el papel diferencial de la intolerancia a la incertidumbre, la evitación cognitiva, la orientación negativa al problema y las meta creencias. Revista Argentina de Clínica Psicológica, 20, 101-108. Recuperado de http://www.clinicapsicologi ca.org.ar/download_file.php?idt $=54 \&$ hax

Rutz, W. (2004). Men's health on the European WHO agenda. Journal of Men's Health and Gender, 1(1), 22-25. https:// doi.org/10.2772/60721

Sánchez, A., \& Vázquez, C. (2012). Sesgos de atención selectiva como factor de mantenimiento y vulnerabilidad de la depresión. Terapia Psicológica, 30(3), 103-117. https://doi.org/10.4067/ S0718-48082012000300010

Scher, C., Ingram, R., \& Segal, Z. (2005). Cognitive reactivity and vulnerability: Empirical evaluation of construct activation and cognitive diatheses in unipolar depression. Clinical Psychology Review, 25(4), 487-510. https:// doi.org/10.1016/j.cpr.2005.01.005

Sheehan, D. V., Lecrubier, Y., Sheehan, K. H., Amorim, P., Janavs, J., Weiller, E., ... Dunbar, G. C. (1998). The Mini-Internactional Neuropsychiatric Interview (M.I.N.I.): The development and validation of a structure diagnostic psychiatric interview for DSM-IV and ICD-10. Journal of Clinical Psychiatry, 59(Supl. 20), 22-33.

Simon, R. W., \& Nath, L. E. (2004). Gender and emotion in the United States: Do men and women differ in self-reports of feelings and expressive behaviors. American Journal of Sociology, 109(5), 1137-1176. https:// doi.org/10.1086/382111

Stoeber, J., \& Otto, K. (2006). Positive conceptions of perfectionism: Approaches, evidence, challenges. Personality and Social Psychology Review, 10, 295-319. Recuperado 
de http://kar.kent.ac.uk/4481/1/Stoeber_\& _Otto_PositiveConceptions_2006.pdf

Strömberg, R., Backlund, L. G., \& Löfvander, M. A. (2010). Comparison between the Beck's Depression Inventory and the Gotland Male Depression Scale in detecting depression among men visiting a drop-in clinic in primary care. Nordic Journal of Psychiatry, 64, 258-264. https:// doi.org/10.3109/08039480903511407

Troister, T. \& Holden, R. (2010). Comparing psychache, depression, and hopelessness in their associations with suicidality: A test of Shneidman's theory of suicide. Personality and Individual Differences, 49(7), 689-693. https:// doi.org/10.1177/0748175612451744

van den Boogaard, M., Verhaak, P. F., van Dyck, R., \& Spinhoven, P. (2011). The impact of causal attributions on diagnosis and successful referral of depressed patients in primary care. Social Science and Medicine, 73(12), 1733-1740. https:// doi.org/10.1016/j.socscimed.2011.09.027

Van de Velde, S., Bracke, P., \& Levecque, K. (2010). Gender differences in depression in 23 European countries. Crossnational variation in the gender gap in depression. Social Science y Medicine, 71(2), 305-313. https://doi.org/10.1016/ j.socscimed.2010.03.035

van Tuijl, L., de Jong, P., Sportel, E., de Hullu, E., \& Nauta, M. (2013). Implicit and explicit self-esteem and their reciprocal relationship with symptoms of depression and social anxiety: A longitudinal study in adolescents. Journal of Behavior Therapy and Experimental Psychiatry, 45, 113-121. https://doi.org/10.1016/j.jbtep.2013.09.007

Viana, M. C., \& Andrade, L. H. (2012). Lifetime prevalence, age and gender distribution and age-of-onset of psychiatric disorders in the São Paulo metropolitan area, Brazil: Results from the São Paulo Megacity Mental Health Survey. Revista Brasileira de Psiquiatria, 34(3), 249-260. https://doi.org/ 10.1016/j.rbp.2012.03.001
Wai, S., \& Tse, T. H. (2009). Relationship among dispositional forgiveness of others, interpersonal adjustment and psychological well-being: Implication for interpersonal theory of depression. Personality and Individual Difference, 46(3), 365-368. https://doi.org/10.1016/j.paid.2008.11.001

Wai, S., Jayne, W., \& Kai-Chung, P. (2011). Motivation for achievement as perceived resource value in social rank theory of depression: A structural equation modeling analysis. Personality and Individual Differences, 50(7), 1034-1038. https:// doi.org/10.1016/j.paid.2011.01.019

Wang, W. T., Hsu, W. Y., Chiu, Y. C., \& Liang, C. W. (2012). The hierical model of social interaction anxiety and depression: the critical roles of fears of evaluation. Journal of Anxiety Disorders, 26(1), 215-224. https:// doi.org/10.1016/j.janxdis.2011.11.004

Watkins, K. E., Hunter, S. B., \& Hepner, K. A. (2011). An effectiveness trial of group cognitive behavioral therapy for patients with persistent depressive symptoms in substance abuse treatment. Archives of General Psychiatry, 68, 577-584. Recuperado de http://www.ncbi.nlm.nih.go v/pmc/articles/PMC3230556/

Weeks, J. W., Jakatdar, T. A., \& Heimberg, R. G. (2010). Comparing and contrasting fears of positive and negative evaluation as facets of social anxiety. Journal of Social and Clinical Psychology, 29, 68-94. https:// doi.org/10.1521/jscp.2010.29.1.68

Welling, H. (2003). An evolutionary function of the depressive reaction: The cognitive map hypothesis. New Ideas in Psychology, $21(2), \quad 147-156$. https://doi.org/10.1016/ S0732-118X(03)00017-5

Wenzel, A., Steer, R., \& Beck, A. T. (2005). Are there any gender differences in frequency of self-reported somatic symptoms of depression? Journal of Affective Disorders, 8(1-3), 177-181. https://doi.org/10.1016/ j.jad.2005.06.009

Wilhelm, K., Boyce, P., \& Brownhill, S. (2004). The relationship between interpersonal sensitivity, anxiety disorders 
and major depression. Journal of Affective Disorders, 79(1-3), 33-41. DOI: 10.1016/ S0165-0327(0200069-1

Zapata, G., \& Giner, M. (2009). La cognición del individuo: reflexiones sobre sus procesos e influencia en la organización. Espacio Abierto, 18(2), 235-256. Recuperado de http://www.redalyc.org/articulo.oa?id= 12211826003

Zeigler-Hill, V. (2011). The connections between self-esteem and psychopathology. Journal of Contemporary Psychotherapy, 41(3), 157-164. https://doi.org/10.1007/ s10879-010-9167-8

\section{Notas}

* Artículo de investigación. 